\title{
Mechanical testing of newly developed biomaterial designed for intra-articular reinforcement of partially ruptured cranial cruciate ligament: ex vivo pig model
}

\author{
Petra Fedorová1, Robert Srnec ${ }^{2}$, Jan Pěnčík ${ }^{3}$, Pavel Schmid ${ }^{3}$, Evžen Amler ${ }^{4,5}$, \\ Lucie Urbanová ${ }^{1}$, Alois Nečas ${ }^{2}$
}
${ }^{1}$ University of Veterinary and Pharmaceutical Sciences Brno, Faculty of Veterinary Medicine, Small Animal Clinic, Department of Surgery and Orthopaedics, ${ }^{2}$ CEITEC - Central European Institute of Technology, Brno, Czech Republic
${ }^{3}$ Brno University of Technology, Faculty of Civil Engineering, Institute of Building Testing, Brno, Czech Republic
${ }^{4}$ Charles University in Prague, $2^{\text {nd }}$ Faculty of Medicine, Institute of Biophysics, Prague, Czech Republic ${ }^{5}$ Academy of Sciences of the Czech Republic, v.v.i., Laboratory of Tissue Engineering, Institute of Experimental Medicine, Prague, Czech Republic

Received August 25, 2013

Accepted January 23, 2014

\begin{abstract}
The study deals with mechanical testing of newly developed material polyethylene terephtalate coated with polycaprolactone nanofibers in combination with biodagradable Hexalon ACL/PCL screws as a new possibility of intra-articular reinforcement of partially ruptured cranial cruciate ligament. Four groups of ex vivo models of pig stifle joints were prepared and tested: a model with intact CCL (group 1), a model with partial CCL rupture (group 2), a model with CCL rupture stabilized with $7 \mathrm{~mm}$ Mersilene ${ }^{\mathbb{R}}$ strip (group 3), and a model with CCL rupture stabilized with $5 \mathrm{~mm}$ PET/PCL biomaterial strip (group 4). The models were loaded in the standing angle of $100^{\circ}$ and the maximum load $(\mathrm{N})$ and the shift $(\mathrm{mm})$ were monitored. The mean maximum peak power and the shift were $1266.0 \pm 146.9 \mathrm{~N}$ and $13.7 \pm 2.5 \mathrm{~mm}$ for group 1 , and $1164.7 \pm 228.2 \mathrm{~N}$ and 1 $6.8 \pm 3.3 \mathrm{~mm}$ for group 2, respectively. In all cases after reaching the maximum load, a tibial fracture occurred but never a CCL rupture. In groups 3 and 4, the initial fixation failure occurred in the mean values of $375.7 \pm 81.5$ and $360.4 \pm 52.0 \mathrm{~N}$, respectively, and with a bigger shift of $52.3 \pm 11.9 \mathrm{~mm}$ and $39.4 \pm 14.6 \mathrm{~mm}$, respectively, compared to group 1. A critical point of failure was the anchoring in the bone. It can be concluded that the PET/PCL substitute in the ex vivo model has mechanically comparable properties with the clinically used Mersilene ${ }^{\circledR}$, and based on its proven ability to carry stem cells it could be appropriate for partially ruptured CCL protection.
\end{abstract}

Knee stabilization, stifle surgery, polycaprolactone nanofibre, polyester, synthetic material, biodegradable screw

Cranial cruciate ligament (CCL) disease is one of the most common orthopaedic conditions of dogs (Christopher et al. 2013). The integrity of the cranial cruciate ligament may be lost due to direct trauma but mostly it is due to degenerative changes of the ligament developing with progression of age of animals (Necas et al. 2000). Modern options include the osteotomy methods that are meant to change the biomechanical properties in the stifle joint (Montavon et al. 2002; Hulse et al. 2010). Various materials have been used for these stabilization procedures and some mechanical studies have already been performed using a variety of methods (Rose et al. 2012).

The aim of the study was to test the mechanical properties of a newly synthesized material made of polyethylene terephtalate coated with polycaprolactone nanofibres (PET/PCL) that should serve as a scaffold for stem cells or bioactive substances as well as a support for the partially damaged CCL. This material was to be employed using a unique intraarticular technique of incorporation into the stifle joint in combination with biodegradable Hexalon ACL/PCL screws.

Phone: +420 541562383

Fax: +420541562 344

E-mail: fedorovap@vfu.cz

http://actavet.vfu.cz/ 


\section{Materials and Methods}

Ex vivo mechanical testing was performed on a defined "bone-ligament-bone" model made from the stifle joint of young slaughter sows after slaughter by cutting a segment of the proximal tibia and distal femur. The segment of the stifle joint was cleaned from the adjacent muscles, joint capsule, lateral and medial meniscus, both collateral ligaments and the caudal cruciate ligament. The model of "tibia-cranial cruciate ligament-femur" was wrapped with gauze moisturized with saline and frozen to $-20{ }^{\circ} \mathrm{C}$ until the moment of testing (Choate et al. 2012). Models were prepared and slowly defrosted for $12 \mathrm{~h}$ at room temperature before the testing. A total of 38 models was tested, divided into 4 groups. Group 1 consisted of models with an intact CCL $(\mathrm{n}=10)$. Group 2 consisted of models with a partial CCL rupture $(n=10)$. Partial iatrogenic CCL rupture was made by cutting the craniomedial portion of CCL with an 11 scalpel blade. Measured data of groups 1 and 2 were used as control groups for comparison with testing groups. In the third group $(n=8)$, commercially produced synthetic substitute polyester $7 \mathrm{~mm}$ Mersilene ${ }^{\circledR}$ (Ethicon) strips were tested. Samples of the newly synthesized biomaterials were tested in the fourth group $(\mathrm{n}=10)$. Individual fibres (Plate VI, Fig. 1) were synthesized from polyethylene terephthalate and coated with polycaprolactone nanofibres (PET/PCL). A unique $5 \mathrm{~mm}$ thick hand-woven rope made of 36 individual threads was made for the testing.

The introduction of all the tested synthetic substitutes was performed in the same manner. A channel with a $5 \mathrm{~mm}$ diameter (Acufex Endoscopic Cannulated Drill Bit $5.0 \mathrm{~mm}$, Smith\&Nephew, USA) was drilled on the origin of CCL on the medial aspect of the lateral femoral condyle and the tunnel outlet was directed at its caudal part. The knot made on the one end of the fibre was inserted into the tunnel and secured with a biodegradable $7 \mathrm{~mm}$ Hexalon ACL/PCL screw with a length of $25 \mathrm{~mm}$ (Hexalon ${ }^{\mathrm{TM}}$, Inion Ltd., Finland) from the side of the articular surface. Then, another $5 \mathrm{~mm}$ wide channel was drilled on the point of insertion of CCL to the tibial crest in the distal direction to the medial aspect of the tibia, and the second tibial channel was drilled approximately $10 \mathrm{~mm}$ distally to the first one in the transversal direction through the proximal metaphysis of the tibia. The biomaterial that was already fixed in the femur was now dragged through the first channel in the tibia and the model of a stifle joint was positioned to the standing angle of $100^{\circ}$ (Miller and Boring 1978). The ligament supplement was fixed tight with $7 \mathrm{~mm}$ Hexalon ACL/PCL screw with a length of $25 \mathrm{~mm}$. In the next step, the free distal end of the rope was additionally fixed with $7 \mathrm{~mm}$ Hexalon ACL/PCL screw with a length of $20 \mathrm{~mm}$.

For the testing, the models were fixed in a special newly constructed handle (Plate VI, Fig. 2). The femur and tibia were firmly fixed in the handle at an angle of $100^{\circ}$ and the tibia was subsequently loaded in the reverse direction with vertical displacement. Power was deduced by the load press FP10 with the measuring range of 0.4-10 kN. For the purpose of testing, the range of $4 \mathrm{kN}$ was chosen and the testing models were loaded with a constant rate of shift. The vertical shift of the load press transom was recorded by inductive track sensor HBM WA-T/50 $\mathrm{mm}$ with the measuring range of 0-50 $\mathrm{mm}$. The magnitude of force and vertical displacement were continuously recorded at a frequency of logging of $5 \mathrm{~Hz}$ (time interval $0.2 \mathrm{~s}$ ) with HBM Spider8 logger. The limit of the maximum load construct $(\mathrm{N})$ before its failure and the shift of the model during the load (mm), were monitored in all test groups. Moreover, in the groups tested with the Mersilene ${ }^{\circledR}$ and PET/PCT substitute, we observed the boundary of beginning failure of the construction detected on the graphic line as the initial decrease of the load $(\mathrm{N})$ during the shift and also quantity of the shift of model for the load (mm) was recorded.

Nonparametric Wilcoxon (Mann-Whitney) test for unpaired data and basic descriptive statistic (Kyplot version 2.0 beta $15-32$ bit) were used for statistical evaluation of results.

\section{Results}

In group 1 with stifles with the intact CCL, a total of 10 models were evaluated. In this group, in all cases after reaching the maximum load a fracture in the proximal tibia occurred but never a CCL rupture. The mean maximum peak power was $1266.0 \pm 146.9 \mathrm{~N}$ (minimal value $1118.4 \mathrm{~N}$ and maximal value $1546.0 \mathrm{~N}$ ). The mean shift of the model was $13.7 \pm$ $2.5 \mathrm{~mm}$ (minimal value $9.6 \mathrm{~mm}$, maximal value $18.4 \mathrm{~mm}$ ). The typical course of the shift of intact CCL depending on an increasing load is shown in Fig. 3. In group 2 with the stifles with partial CCL rupture, a total of 10 models were evaluated, too. In all cases after reaching the maximum load a fracture in the proximal tibia occurred but no CCL rupture. The mean maximum peak power was $1164.7 \pm 228.2 \mathrm{~N}$ (minimal value $788.6 \mathrm{~N}$ and maximal value $1462.7 \mathrm{~N}$ ). The mean shift of the model imitating the partial CCL rupture was $16.8 \pm 3.3 \mathrm{~mm}$ (minimal value $11.9 \mathrm{~mm}$ and maximal value $22.7 \mathrm{~mm}$ ). Comparing group 1 with group 2, no significant difference $(P>0.05)$ was found in the maximum load achieved but in group 2, a slightly greater $(P \leq 0.05)$ shift of the model was observed. In the group of stifle models with CCL rupture stabilized with commercially available polyester $7 \mathrm{~mm}$ Mersilene ${ }^{\circledR}$ (Ethicon) strip (group 3), a total of 8 models was evaluated. In group 3 six cases after reaching the 


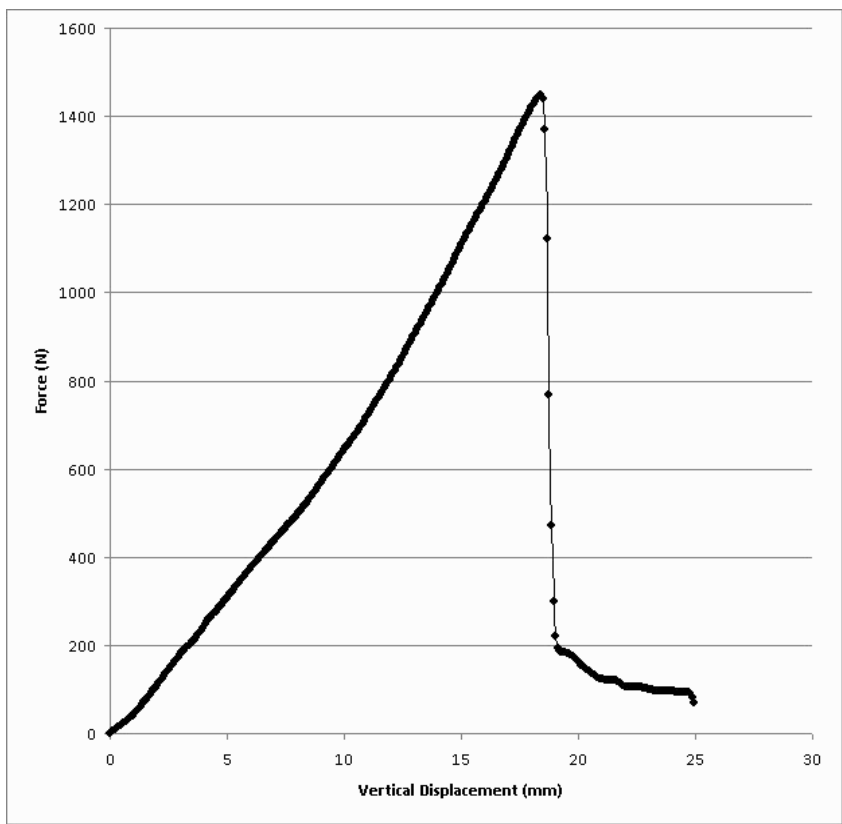

Fig. 3. Graph of the mechanical load depending on the degree of the stifle joint model deformation in the control group without cranial cruciate ligament

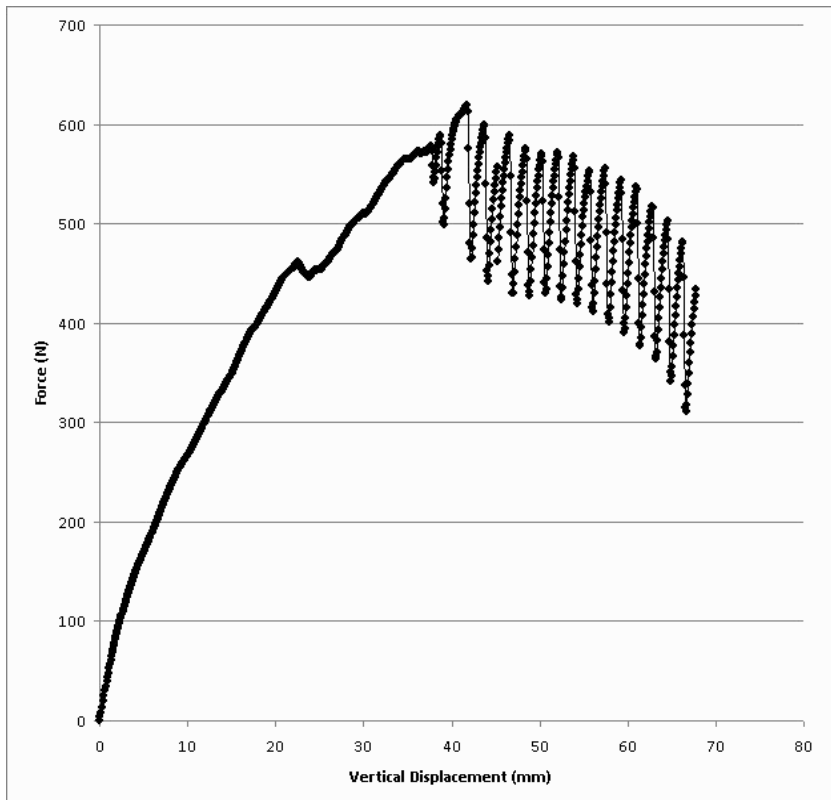

Fig. 4. Graph of the mechanical load depending on the degree of the stifle joint model deformation in the $7 \mathrm{~mm}$ Mersilene ${ }^{\circledR}$ polyester strip substitute 


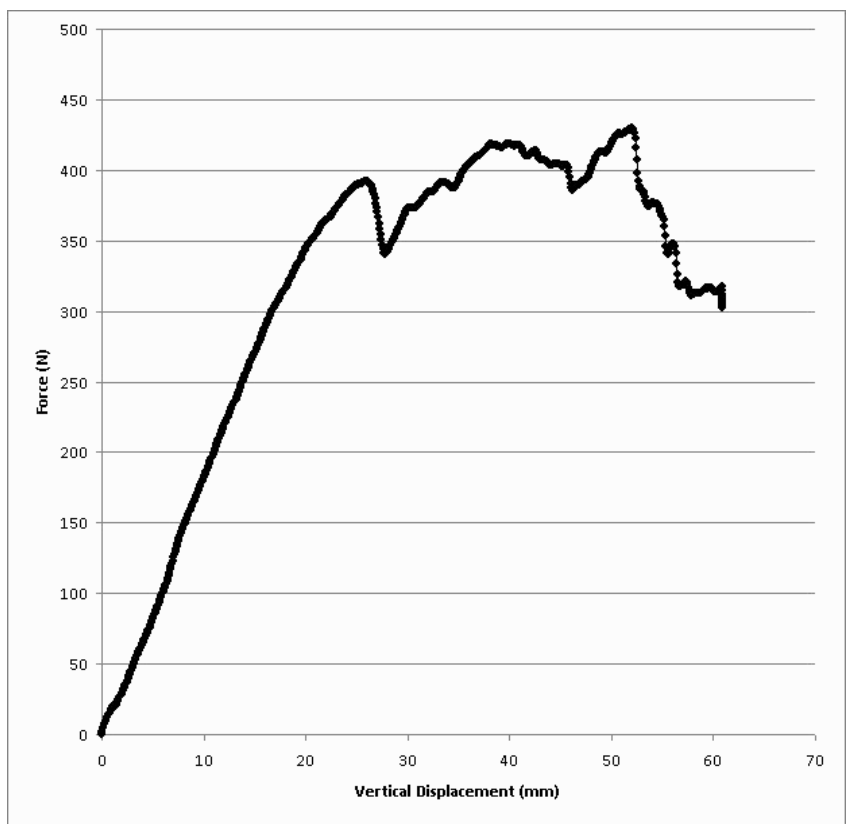

Fig. 5. Graph of the mechanical load depending on the degree of the stifle joint model deformation in the polyethylene terephtalate coated with polycaprolactone substitute

maximum load the substitute released from its anchor in tibia and in two cases it released from femur. The polyester Mersilene ${ }^{\circledR}$ strip was not broken in any case. The mean maximum peak power was $553.3 \pm 118.5 \mathrm{~N}$ (minimal value $389.6 \mathrm{~N}$ and maximal value $685.2 \mathrm{~N}$ ). The mean shift was $52.3 \pm 11.9 \mathrm{~mm}$ (minimal value $35.2 \mathrm{~mm}$ and maximal value $71.3 \mathrm{~mm}$ ). Compared to group 1 , a highly significant $(P \leq 0.001)$ difference in the maximum load was achieved and the shift was observed. A typical course of the shift of the Mersilene ${ }^{\circledR}$ substitute depending on the increasing load is shown in Fig. 4.

In group 4 with stifle models with CCL rupture stabilized with polyethylene terephtalate coated with polycaprolactone nanofibres (PET/PCL), a total of 9 models was tested. One model was eliminated from the testing because of substitute failure caused by an unravelling of the threads. In 7 cases, after reaching the maximum load limit the substitute was released from the tibia and in two cases the PET/PCL substitute of the CCL was broken. The mean maximum power peak was $397.4 \pm 58.9 \mathrm{~N}$ (minimal value $320.8 \mathrm{~N}$ and maximal value $479.4 \mathrm{~N}$ ). The mean shift of the model was $39.4 \pm 14.6 \mathrm{~mm}$ (minimal value $18.1 \mathrm{~mm}$ and maximal value $64.4 \mathrm{~mm}$ ). Compared to group 1 , a highly significant difference $(P \leq$ $0.001)$ in the maximum load achieved and deformation was observed. A typical course of the shift of the PET/PCL substitute of CCL depending on the increasing load is shown in Fig. 5. When comparing models with polyester $7 \mathrm{~mm}$ Mersilene ${ }^{\circledR}$ strip CCL and models with PET/PCL, a significant difference $(P \leq 0.01)$ in the maximum load reaching was observed but there was not significant difference $(P>0.05)$ in the magnitude of the shift of the created construct. However, if the comparison is not made right after the reaching of maximum load values but at the first load drop during the shift of the model (Figs 4 and 5), there were no significant differences $(P>0.05)$ in the values of the load or in the shift of the model for the load between the two groups. The mean strength in reaching this 
stage of incipient failure of polyester substitute was $375.7 \pm 81.5 \mathrm{~N}$ (minimal value 271.8 $\mathrm{N}$ and maximal value $485.0 \mathrm{~N}$ ) and for PET/PCL substitute $360.4 \pm 52.0 \mathrm{~N}$ (minimal value $290.3 \mathrm{~N}$ and maximal value $440.1 \mathrm{~N}$ ). The mean shift of the model deducted at this point of initial failure in the model with the polyester substitute was $19.8 \pm 4.9 \mathrm{~mm}$ (minimal value $13.3 \mathrm{~mm}$ and maximal value $24.7 \mathrm{~mm}$ ) and in models with the PET/PCL substitute $21.4 \pm 4.8 \mathrm{~mm}$ (minimal value $14.5 \mathrm{~mm}$ and maximal value $28.2 \mathrm{~mm}$ ).

\section{Discussion}

For the purposes of ex vivo testing, we chose a CCL substitute model created from the knee joint of pigs. Models using pigs are used also in the latest biomechanical study in knee joints (Ehrensberger et al. 2013). CCL damage is caused by a complex of causal biological and biomechanical factors (Cook 2010; Griffon 2010). This fact is also supported by our test results of the control groups when after reaching the limit maximum load no rupture of CCL was observed but fractures of the proximal tibial growth plate of adolescent pigs were found. The direction of force during the testing should as much as possible reflect the physiological condition of loading the CCL or its substitute. In pigs, we respected the standing angle of $100^{\circ}$ (Miller and Boring 1978) and fixation of the model kept the angle between the longitudinal axis of the femur and tibia during the test. In dogs, the standing angle is usually bigger (about $135^{\circ}$ ) and a study describing this value respects it according to the anchoring points of substitutes (Choate et al. 2012).

A number of works deal with the mechanical properties of materials used as a substitute for CCL (Burgess et al. 2010; Ledecky et al. 2012; Rose et al. 2012), however, from the evaluation of our results we can see that the weak point is especially in the anchoring of the substitute and in the properties of this connection. Therefore, we consider it important to evaluate the whole construction of fixation of the substitute to the bone rather than the material itself. In the testing of both types of CCL substitutes on a graph an evident drop is visible during the load just before reaching the maximum value. This is probably caused by tightening the knot of the material in the bone tunnel towards to the thread of the ACL/PCL screw. This value is already considered as the beginning of the construction failure and it is comparable in both types of stabilizing material.

However, further development of the graph is different. In the case of the Mersilene ${ }^{\circledR}$ polyester substitute there is an evident major load increase and just then a total failure and slipping of the smooth material along the screw thread (oscillation of the load values is presented in Fig. 4) and also a major stretching of the construction. In the model with the PET/PCL substitute, after the initial failure very fast reaching of the maximum and a rapid release of the substitute occur. This different behaviour is due to different material properties and viscous-elastic connection with bone. Besides, the biodegradable screw is often used in human medicine for the fixation of hamstring autograft (Jarvela 2007) and a synthetic material is not fixed so effectively. Models of the knee joint with a synthetic CCL substitute (PET/PCL and Mersilene ${ }^{\circledR}$ ) are gradually tightened during the test, the load increase is slower, and the degree of shift significantly higher compared to the control group. The mean level of maximum load at the first failure of the synthetic substitute was $360 \mathrm{~N}$ and $375 \mathrm{~N}$. Compared to the control group the values are significantly lower ; however, for the needs of fixation in dogs we considered it sufficient. Published data present the load of CCL when walking and idle for a dog of about $50 \mathrm{~N}$, and the load in the range from $400 \mathrm{~N}$ to $600 \mathrm{~N}$ (Caporn and Roe 1996; Choate et al. 2012). At the same time the mechanical support of the intact part of CCL is expected. In the case of PET/PCL material, it was certainly sufficient compared to the material commonly used for extra-articular stabilization (Mersilene ${ }^{\mathbb{R}}$ ). Biocompatibility of the PET/PCL substitute was included in other studies. Other sources describe other CCL substitutes with the function 
of carrier silk-collagen based scaffolds (Panas et al. 2009). The PET/PCL substitute in combination with a biodegradable screw provides sufficient mechanical support for preservation of properties of the carrier with a wide range of applications. The anchoring of the substitute in the bone appears as a limiting point. In our opinion, improvement can be achieved with better processing of PET/PCL fibres in the proper substitute and a better method of its anchoring. Before clinical use it is necessary to provide in vivo tests using an animal model.

\section{Acknowledgements}

This study was supported by Ministry of Education, Youth and Sport of the Czech Republic (Research project IGA VFU No. 39/2012/FVL) and by the project "CEITEC - Central European Institute of Technology" (CZ.1.05/1.1.00/02.0068) from the European Regional Development Fund.

\section{References}

Burgess R, Elder S, McLaughlin R, Constable P 2010: In vitro biomechanical evaluation and comparison of FiberWire, FiberTape, OrthoFiber, and Nylon leader line for potential use during extraarticular stabilization of canine cruciate deficient stifles. Vet Surg 39: 208-215

Caporn TM, Roe SC 1996: Biomechanical evaluation of the suitability of monofilament nylon fishing line and leader line for extra-articular stabilization of the cranial cruciate deficient stifle. Vet Comp Orthop Traumatol 9: $126-133$

Choate ChJ, Pozzi A, Lewis DD, Hudson CC, Conrad BP 2012: Mechanical properties of isolated loops of nylon leader material, polyethylene cord, and polyethylene tape and mechanical properties of those materials secured to cadaveric canine femurs via lateral femoral fabellae, toggles placed through bone tunnels, or bone anchors. Am J Vet Res 73: 1519-1529

Christopher SA, Beetem J, Cook JL 2013: Comparison of long-term outcomes associated with three surgical techniques for treatment of cranial cruciate ligament disease in dogs. Vet Surg 42: 329-334

Cook JL 2010: Cranial cruciate ligament disease in dogs: Biology versus biomechanics. Vet Surg 39: 270-277

Ehrensberger M, Hohman DW, Duncan K, Howard C, Bisson L 2013: Biomechanical comparison of femoral fixation devices for anterior cruciate ligament reconstruction using a novel testing method. Clin Biomech 28: 193-198

Griffon DJ 2010: A review of the pathogenesis of canine cranial cruciate ligament disease as a basis for future preventive strategies. Vet Surg 39: 399-409

Hulse D, Hyman W, Beale B, Saunders B, Peycke I, Hosgood G 2010: Determination of isometric points for placement of a lateral suture in treatment of the cranial cruciate ligament deficient stifle. Vet Comp Orthop Traumatol 23: 163-167

Jarvela T 2007: Double-bundle versus single-bundle anterior cruciate ligament reconstruction: a prospective, randomize clinical study. Knee Surg Sports Traumatol Arthrosc 15: 500-507

Ledecky V, Knazovicky D, Badida M, Dulebova L, Hluchy M, Hornak S 2012: Mechanical testing of orthopaedic suture material and a crimp clamp system for the extracapsular stabilisation of canine cruciate-deficient stifles. Vet Med 57: 597-602

Miller HW, Boring G 1978: X-Ray analysis of structural soundness in swine. National Swine Improvement Federation. Proceedings of the annual conference 1978, Mississippi State University, p. 35

Montavon PM, Damur DM, Tepic S 2002: Advancement of the tibial tuberosity for the treatment of cranial cruciate deficient canine stifle. Proceedings of the 1st World Veterinary Orthopedic Congress, Munich, Germany, $152 \mathrm{p}$.

Necas A, Zatloukal J, Kecova H, Dvorak M 2000: Predisposition of dog breeds to rupture of the cranial cruciate ligament. Acta Vet Brno 69: 305-310

Panas E, Gatt CJ, Dunn MG 2009: In vitro analysis of a tissue-engineered anterior cruciate ligament scaffold. $35^{\text {th }}$ Annual northeast bioengineering conference, Cambridge MA, 03-05 April 2009, pp. 286-287

Rose ND, Goerke D, Evans RB, Conzemius MG 2012: Mechanical testing of orthopedic suture material used for extra-articular stabilization of canine cruciate ligament-deficient stifles. Vet Surg 41: 266-272 
Plate VI

Fedorová P. et al.: Mechanical testing ... pp. 55-60

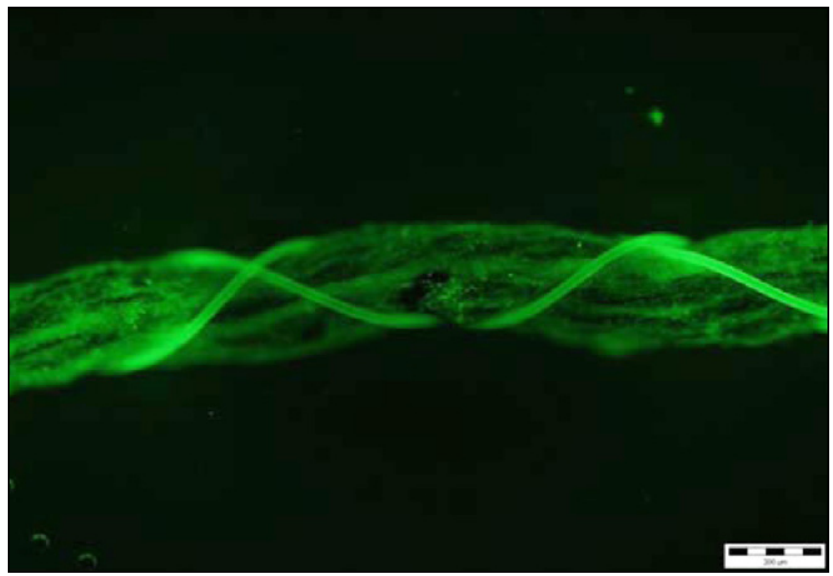

Fig. 1. Individual fibres of polyethylene terephthalate coated with nanofibres of polycaprolactone - material fitted with fibroblasts. Picture from an optical microscope, magnification $\times 100$, stained with ethidium bromide

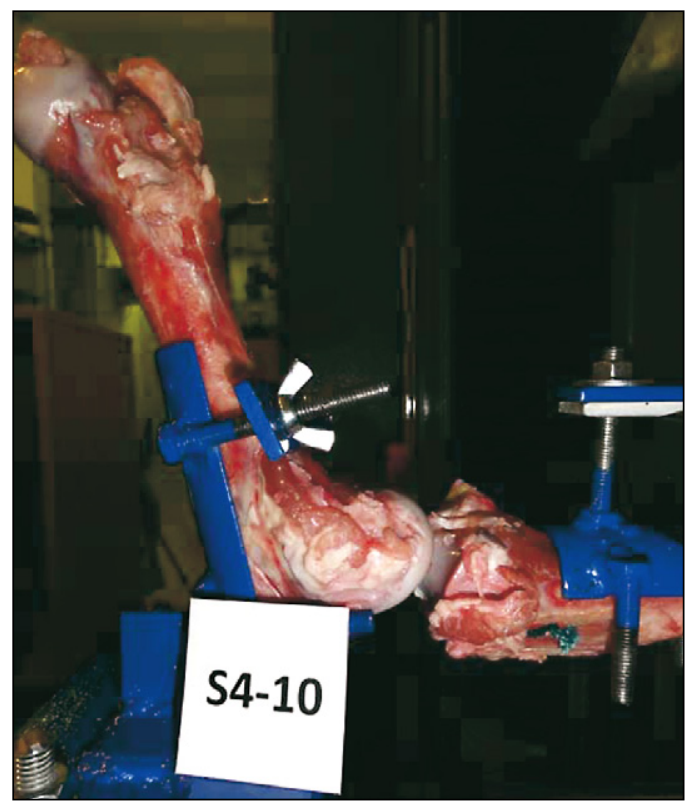

Fig. 2. Newly constructed jig for mechanical testing of cranial cruciate ligament pig model 